\title{
Detection Capability and CFAR Loss Under Fluctuating Targets of Different Swerling Model for Various Gamma Parameters in RADAR
}

\author{
Md. Maynul Islam, Mohammed Hossam-E-Haider \\ Department of Electrical, Electronic and Communication Engineering \\ Military Institute of Science and Technology \\ Dhaka, Bangladesh
}

\begin{abstract}
Target detection of RADAR deals with different and manifold problems over few decades. The detection capability is one of the most significant factors in RADAR system. The main aim of detection is to increase probability of detection while decreasing rate of false alarm. The threshold of detection is modified as a function of the receiver noise level to keep a fixed rate of false alarm. Constant False Alarm Rate (CFAR) processors are used to maintain the amount of false alarm under supervision in a diverse background of interference. In Signal to Noise Ratio (SNR) level, a loss can be occurred due to CFAR processor. Gamma function is used to determine the probability of false alarm. It is assumed in adaptive CFAR that the interference distribution is familiar here. This type of CFAR also approximates the unknown parameters connected with various interference distributions. CFAR loss depends on gamma function. Incomplete gamma function plays an important role in maintaining threshold voltage as well as probability of detection. Changing the value of gamma function can improve the probability of detection for various Swerling Models which are proposed here. This paper has also proposed a technique to compare various losses due to CFAR in terms of different gamma function in presence of different number of pulses for four Swerling Models.
\end{abstract}

Keywords-Swerling model; Constant False Alarm Rate (CFAR) loss; false alarm; gamma function; probability of detection

\section{INTRODUCTION}

In presence of non-stationary background noise (or noise plus clutter) the detection of radar return signals becomes complicated. Function of time can represent a radar target depending on the huge number of real targets whose return changes in magnitude from low to high. Probability of detection in radar depends on many parameters, incomplete gamma function is one of them. Varying the value of gamma parameter, the detection capability can be improved. In Constant False Alarm Rate, the measurement of the noise power levels from the leading and the trailing reference windows are dependent on the Cell Averaging (CA) technique [1]-[4]. The efficiency of CA detector is evaluated in the situations when the operating environment is perfect and when it includes some of fallacious targets along with the target of interest. The primary and the secondary targets are considered to be fluctuating in terms of four Swerling models. The theoretical results show that for various False Alarm rates the probability of detection will be different for various gamma parameter. Four types of Swerling Model have various CFAR rate for changing number of pulses in presence of different gamma parameters.

\section{SYSTEM MODEL}

When only noise present in the radar, the probability of false alarm $P_{f a}$ is defined when a sample exceed the threshold voltage $V_{T}$. The detection probability $\mathrm{P}_{\mathrm{D}}$ is the probability that a sample can surpass the threshold voltage having noise plus signal. It can be written as,

$$
P_{D}=\int_{V_{T}}^{\infty} \frac{r}{\Psi^{2}} I_{o}\left(\frac{r A}{\Psi^{2}}\right) \exp \left[-\left(r^{2}+A^{2}\right) / 2 \Psi^{2}\right] d r
$$

Where $r$ is the envelope of the threshold voltage, $A$ is the amplitude of the return signal with variance of noise $\Psi^{2}$. For a radar signal of sine waveform having amplitude $A$, the power of the signal will be $\mathrm{A}^{2} / 2$.

The Chi-square distribution is applied to a wide range of targets, its $p d f$ can be written as,

$$
f(\sigma)=\frac{m}{\Gamma(m) \sigma_{a v g}}\left(\frac{m \sigma}{\sigma_{\text {avg }}}\right)^{m-1} e^{-\frac{m \sigma}{\sigma_{a v g}}}
$$

Where, $\Gamma(m)$ is the gamma function of argument $\mathrm{m}$ and $\sigma_{a v g}$ is the average value. As the degree gets larger the distribution corresponds to constrained Radar Cross Section (RCS) values. The limit $\mathrm{m}$ tends to $\infty$ corresponds to a constrained RCS target.

Detection of signals threshold is constantly balanced as a function of the receiver noise level in different cases to maintain a constant false alarm rate [5]. In Signal to Noise Ratio (SNR) level a loss of $1 \mathrm{~dB}$ can be occurred due to CFAR processor.

In order to maintain a fixed predetermined probability of false alarm, the threshold of detection is calculated. A relationship between the threshold value $\mathrm{V}_{\mathrm{T}}$ and the probability of false alarm $\mathrm{P}_{\mathrm{fa}}$ can be shown as:

$$
V_{T}=\sqrt{2 \Psi^{2} \ln \left(\frac{1}{P_{f a}}\right)}
$$

If the noise power $\Psi^{2}$ is assumed to be constant, then a fixed threshold can satisfy the above equation. However, due to many reasons this condition is rarely true. In order to maintain a constant probability of false alarm the threshold value must 
be continuously updated based on the estimates of the noise variance. The method of continuously changing the threshold value to maintain a fixed probability of false alarm is known as Constant False Alarm Rate (CFAR) [6].

The Swerling models were introduced to model a variety of target reflections that occur over the radar integration interval. In Swerling model I \& II where the signal amplitudes are fully correlated over the incoherent integration interval but are independent from one integration interval to the next. In Swerling model II \& IV the signal amplitudes are uncorrelated from pulse to pulse throughout the integration interval [7].

The probability of false alarm corresponding to a fixed threshold was derived earlier. When CA-CFAR is implemented, then the probability of false alarm can be derived from the conditional false alarm probability, which is averaged over all possible values of the threshold in order to achieve an unconditional false alarm probability. The conditional probability of false alarm when $y=V_{T}$ can be written as [6]

$$
P_{f a}\left(V_{T}\right)=e^{-\left(\frac{y}{2 \Psi^{2}}\right)}
$$

As a result, unconditional probability of false alarm is [8]

$$
P_{f a}=\int_{0}^{\infty} P_{f a}(y) f(y) d y
$$

Where, $f(y)$ is the $p d f$ of the threshold value.

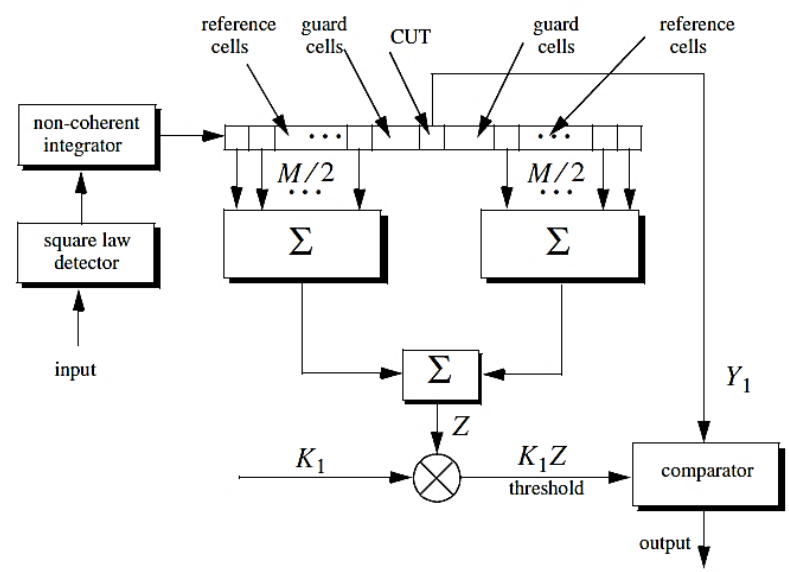

Fig. 1. Conventional CA-CFAR with non-coherent integration.

Practically, CFAR averaging is often implemented after non-coherent integration and the output of each reference cell is the sum of squared envelopes (Fig. 1). It follows that the total number of summed reference samples is $\mathrm{Mn}_{\mathrm{p}}$. The output $\mathrm{Y}_{1}$ is also the sum of $\mathrm{n}_{\mathrm{p}}$ squared envelopes.

When noise alone is present in the Cell Under Test (CUT), $\mathrm{Y}_{1}$ is random variable whose $p d f$ is a gamma distribution with $2 n_{p}$ degrees of freedom. Additionally, the summed output of the reference cells is the sum of $\mathrm{Mn}_{\mathrm{p}}$ squared envelopes. Thus, $\mathrm{Z}$ is also a random variable which has a gamma $p d f$ with $2 \mathrm{Mn}_{\mathrm{p}}$ degrees of freedom [9].

The probability of false alarm is then equal to the probability that the ratio $\mathrm{Y}_{1} / \mathrm{Z}$ exceeds the threshold. More precisely,

$$
P_{f a}=\operatorname{Prob}\left\{\frac{Y_{1}}{Z}>K\right\}
$$

In target detection, threshold $\mathrm{V}_{\mathrm{T}}$ can be determined from probability of false alarm, $\mathrm{P}_{\mathrm{fa}}$. For any number of pulses and non-coherent integration DiFranco \& Rubin give a standard form relating threshold \& probability of false alarm [9].

$$
P_{f a}=1-\Gamma_{I}\left(\frac{V_{T}}{\sqrt{n_{p}}}, n_{p}-1\right)
$$

Where, $\Gamma_{I}$ is used to denote the incomplete gamma function and it can be expressed as [9].

$$
\Gamma_{I}\left(\frac{V_{T}}{\sqrt{n_{p}}}, n_{p}-1\right)=\int_{0}^{V_{T} \sqrt{n_{p}}} \frac{e^{-\gamma} \gamma^{n_{p}-1-1}}{\left(n_{p}-1-1\right) !} d \gamma
$$

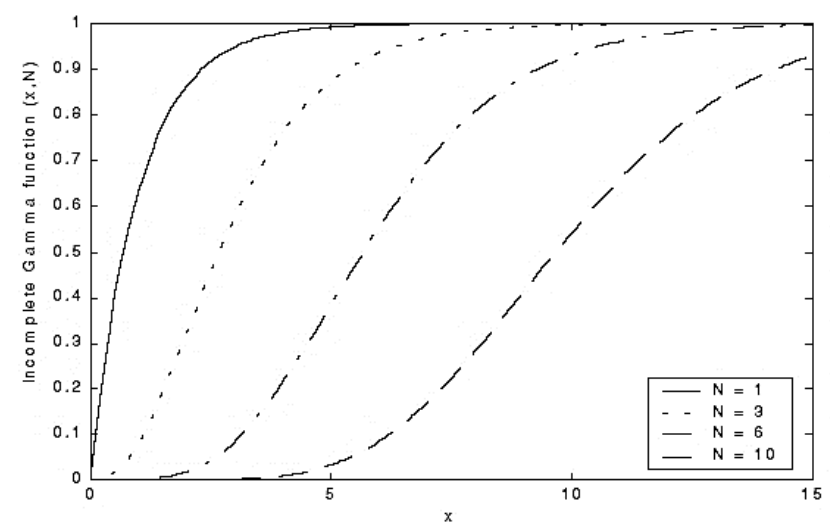

Fig. 2. The incomplete gamma function for different values of $\mathrm{N}$.

Fig. 2 shows the behaviour of incomplete gamma function with respect to independent variable which can be varied due to $x$ and $N$ [10].

\section{SimUlation}

For different Swerling Model Simulation has been carried out in terms of fluctuating target. Conventional Cell Averaging CFAR has been used here. Probability of detection is different for different false alarm rate if the Signal to Noise Ratio (SNR $\mathrm{dB}$ ) varies firmly. Due to loss of constant false alarm rate (CFAR) [11]-[15], probability of detection can be changed in radar detection for fluctuating target. For every model of Swerling, CFAR loss has been simulated and compared. Detection probability of target detection can be found in terms of Signal to Noise Ratio (SNR). From probability of false alarm, loss occurred due to Constant false alarm rate was calculated and compared with respect to gamma function for different swerling model on fluctuating target.

\section{RESULT AND DISCUSSION}

In this paper, comparison has been shown for CFAR loss vs gamma function for four types of Swerling model. From Fig. 3 it is seen that the value of CFAR loss is decreasing with increasing of gamma function for Swerling model I. In this case CFAR loss can be reduced if the number of pulse is comparatively less. 


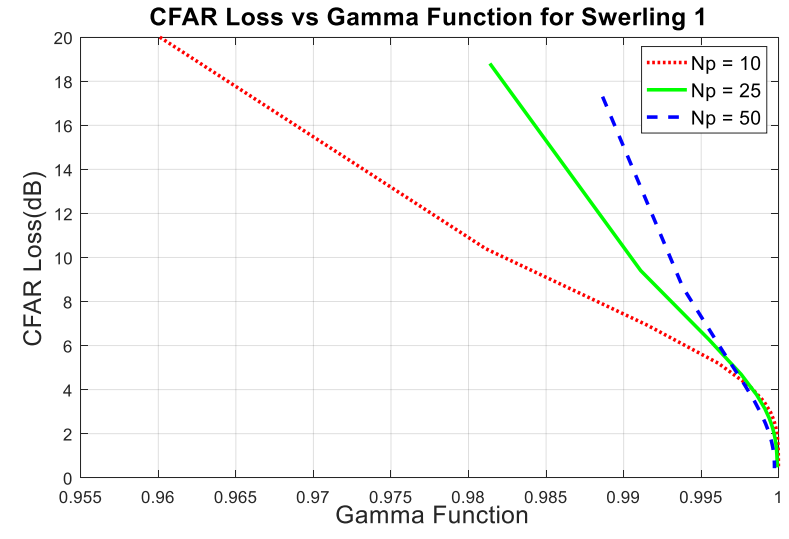

Fig. 3. CFAR Loss vs Gamma Parameter for Swerling Model I.

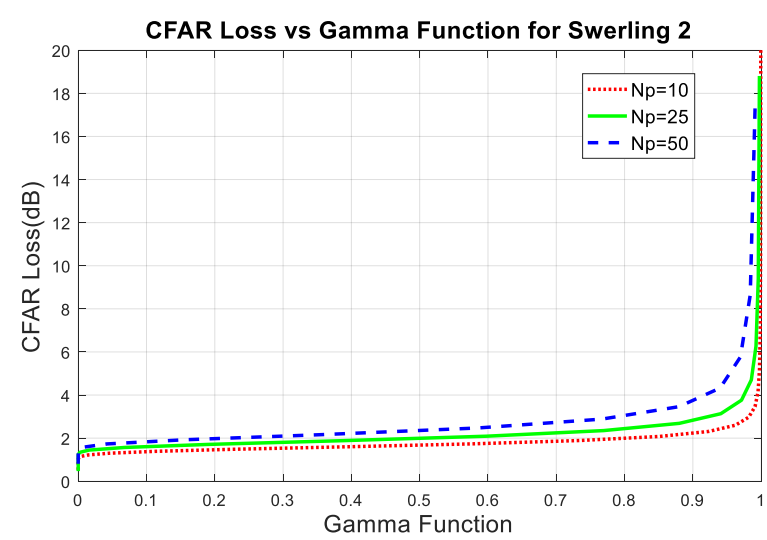

Fig. 4. CFAR Loss vs Gamma Parameter for Swerling Model II.

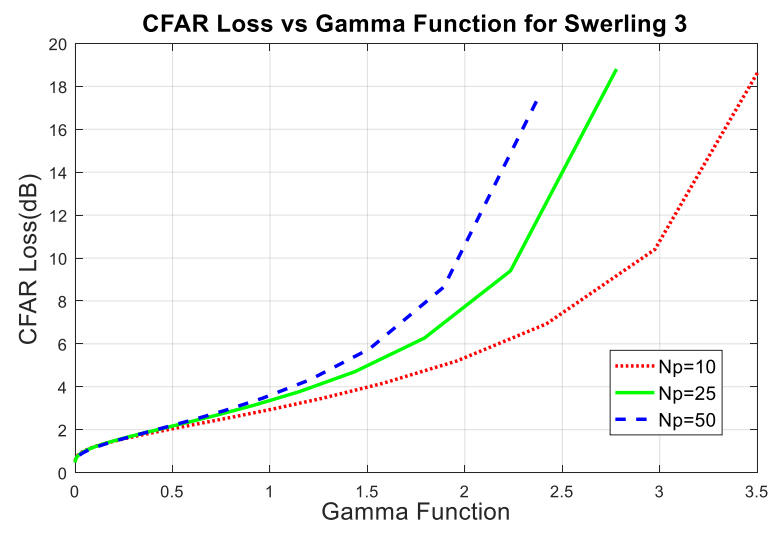

Fig. 5. CFAR Loss vs Gamma Parameter for Swerling Model III

From Fig. 4 it is clear that the value of CFAR loss is sharply increasing after a certain period of gamma function for Swerling model II. The curve of CFAR loss slightly varies from others for different number of pulses.

It is shown in Fig. 5 that CFAR loss is increased if the value of gamma function increases for Swerling model III. CFAR loss is comparatively low for less number of pulses.

For Swerling model IV from Fig. 6 it is seen that the curve of CFAR loss shows rapid response in terms of gamma function. For a small value of gamma CFAR loss can be increased sharply upto $20 \mathrm{~dB}$.

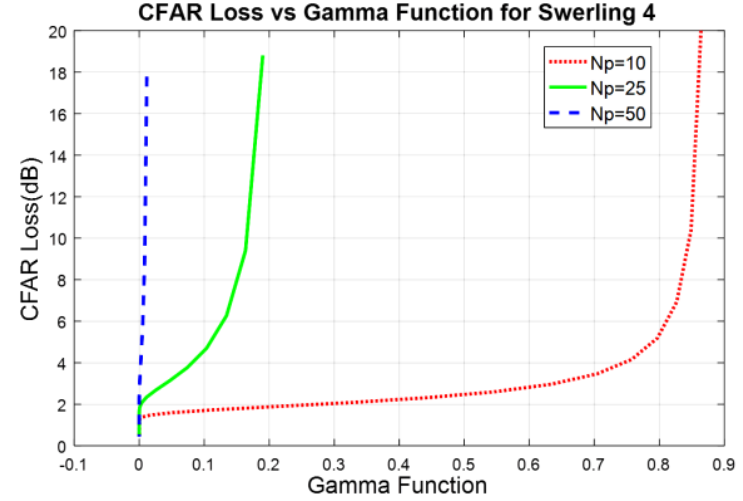

Fig. 6. CFAR Loss vs Gamma Parameter for Swerling Model IV.

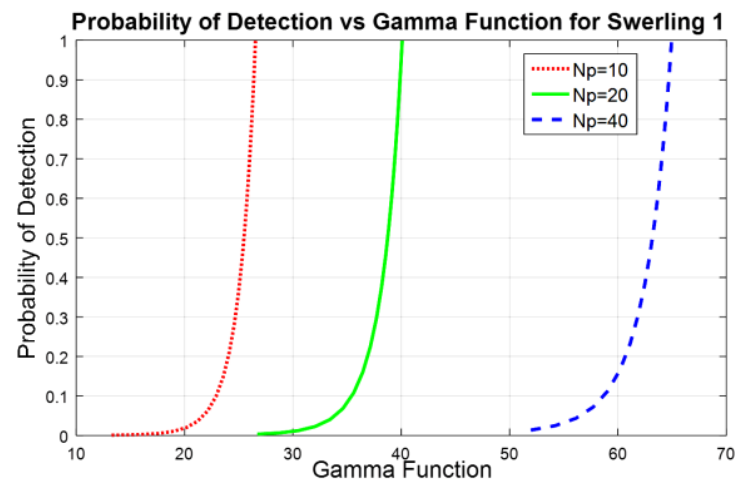

Fig. 7. Pd vs Gamma Parameter for Swerling Model I.

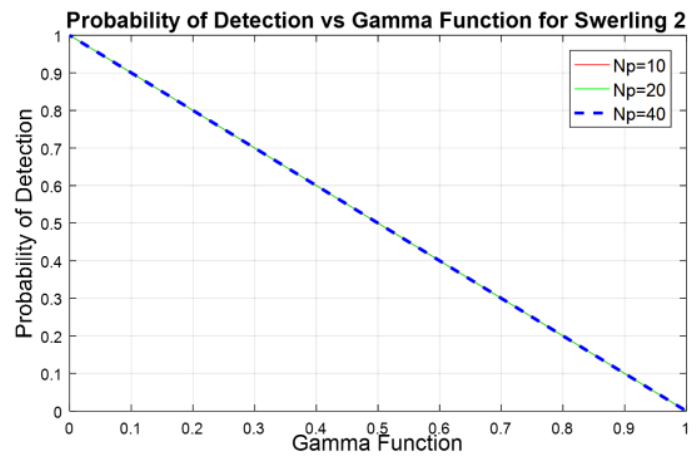

Fig. 8. Pd vs Gamma Parameter for Swerling Model II

Probability of detection also depends on gamma parameter. For Swerling model 1 detection capability of radar increases rapidly for a little change of gamma function (Fig. 7). For different number of pulses the range of gamma parameter is different.

The fluctuation of targets is independent from pulse to pulse rather than from scan to scan for Swerling model 2. From Fig. 8 it is clear that probability of detection decreases linearly with increasing gamma function. It happens for any number of pulse.

Probability of detection also decreases with increasing gamma function in Swerling model 3. But the behavior of this curve is almost linear in nature. If the number of pulse is higher, the detection capability improves for any values of gamma parameter which is shown in Fig. 9. 


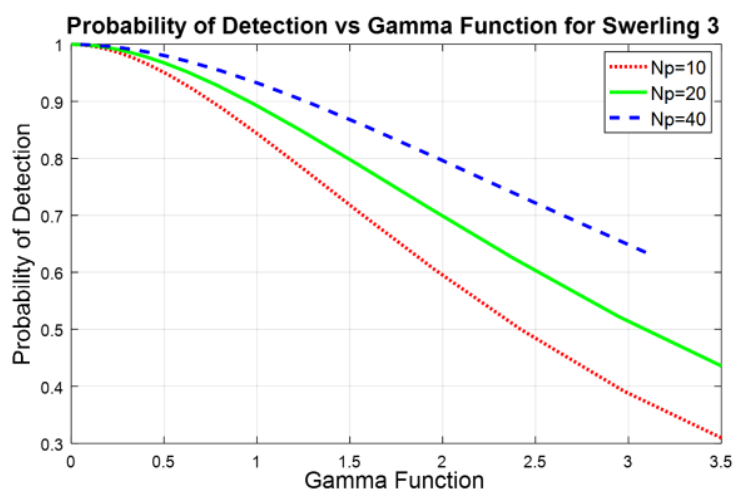

Fig. 9. Pd vs Gamma Parameter for Swerling Model III.

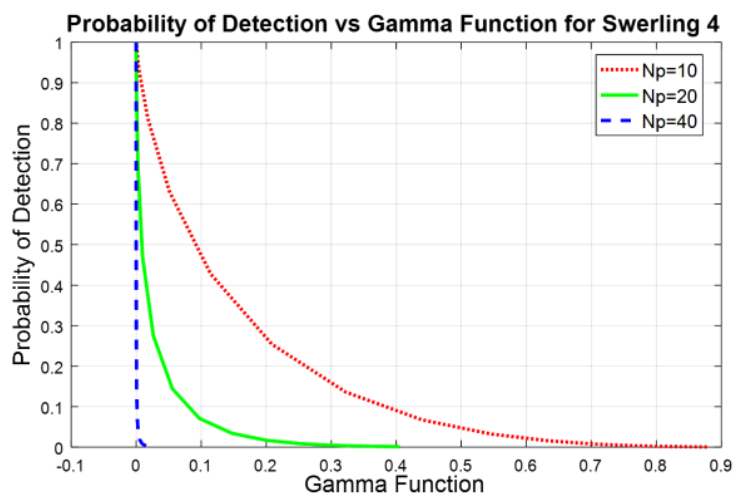

Fig. 10. Pd vs Gamma Parameter for Swerling Model IV.

For Swerling model 4, the detection capability rapidly decreases from its highest value with incrasing gamma parameter. For different number of pulses the curve shows various behaviours which are mentioned in Fig. 10.

It is clear from the above figures that there happens more CFAR loss for Swerling model II, III \& IV if the value of gamma function is increased. For Swerling model I, CFAR loss is less for increasing value of gamma function. Again, gamma function is related to probability of detection in RADAR. At low value of gamma, the CFAR loss is minimum for Swerling model III. It is clear that Swerling model III has the second highest detection capability where the loss due to Constant False Alarm Rate (CFAR) is lower. For Chi square distribution used in probability of detection, gamma is inversely proportional to the pdf. For Swerling model II, III \& IV the probability of detection decreases with increasing gamma parameter. For better detection capability, the value of gamma function should be kept as low.

\section{CONCLUSION}

This paper presents an analytical method for comparison of CFAR loss for various value of gamma function \& method of improving detection capability in RADAR technology. Differences among four Swerling model have been simulated in case of fluctuating target. It is observed that lower CFAR loss can give better accuracy in target detection. For probability of detection lower gamma function is also desirable. It has been revealed that decreasing the value of gamma as well as increasing Cell Array can be the better solution where targets are fluctuated. In future analysis, comparison between CFAR Loss and Cell Array can be a new dimension of this research. Due to inherent nature of coherent and non-coherent integration, the proposed system is flexible and easy to implement.

\section{REFERENCES}

[1] G. Gigli and G. A. Lampropoulos, "A new maximum likelihood generalized gamma CFAR detector," in Geoscience and Remote Sensing Symposium, 2002, Toronto, Ontario, Canada, 2002.

[2] C. J. Willis, "Target modeling for SAR image simulation," The International Society for Optical Engineering, vol. 9243, Oct. 2014.

[3] B. Thomas and S. L. Donnie, "Improved RCS model for censored Swerling III and IV target models," in IEEE Aerospace Conference Proceedings, 2013.

[4] E. M. Mohamed, "Analytical performance evaluation of adaptive detection of fluctuating radar targets," Radioelectronics and Communications Systems, vol. 56(7), pp. 321-334, Jul. 2013.

[5] K. Lingjiang, C. Guolong, Y. Xiaobo, and W. Bing, "Constant false alarm rate performance prediction for non-independent and nonidentically distributed gamma fluctuating targets," IET Radar Sonar Navigation, vol. 10(5), Oct. 2015.

[6] Mahafza, Bassem, and Atef Elsherbeni. "Radar Detection", Electrical Engineering Handbook, 2009.

[7] D.A. Shnidman, "Expanded swerling target models", IEEE Transactions on Aerospace and Electronic Systems, 2003.

[8] Mahafza, "Radar Detection", Radar Systems Analysis and Design Using MATLAB, 2000.

[9] B. R. Mahafza, "Radar Systems Analysis and Design Using MATLAB." CHAPMAN \& HALL/CRC, 2000.

[10] Mahafza, "Target Detection and Pulse Integration", Radar Signal Analysis and Processing Using MATLAB, 2008.

[11] B. El Mashade, Mohamed. "Partially-Correlated $\chi^{2}$ Targets Detection Analysis of GTM-Adaptive Processor in the Presence of Outliers", International Journal of Image Graphics and Signal Processing, 2014.

[12] Md. Maynul Islam, Md. Roman Sarker, Md. Tanjilul Alam, and Mohammed Hossam-E-Haider, "Comparison of analog and digital pulse compression technique and reduction of side lobes using transversal filter", International Conference on Electrical Engineering and Information \& Communication Technology, 2014.

[13] W. Bing, C. Guolong, K. Lingjiang, and Y. Xiaobo, "Performance prediction of the CFAR detector for generalized Swerling-Chi fluctuating targets," in IEEE National Radar Conference Proceedings, 2013, vol. 52(1), pp. 1-5.

[14] E'. Magraner, N. Bertaux, and P. Re'fre' gier, "A new CFAR detector in gamma-distributed nonhomogeneous backgrounds," presented at the 16th European Signal Processing Conference (EUSIPCO 2008), Lausanne, Switzerland, 2008.

[15] L. H., Z. S., and Y. J., "An integrated target detection and tracking algorithm with constant track false alarm rate," Journal of European Industrial Training, May 2016. 